\title{
Multi-Perspective Tolerance Evaluation of Bacillus Calmette-Guerin with Interferon in the Treatment of Non-Muscle Invasive Bladder Cancer
}

\author{
Ryan L. Steinberg ${ }^{\mathrm{a}}$, Lewis J. Thomas ${ }^{\mathrm{b}}$, Sarah L. Mott ${ }^{\mathrm{c}}$ and Michael A. O’Donnell ${ }^{\mathrm{c}, \mathrm{d}, *}$ \\ ${ }^{a}$ Department of Urology, University of Texas Southwestern, Dallas, TX, USA \\ ${ }^{\mathrm{b}}$ Department of Urology, Cleveland Clinic, Cleveland, OH, USA \\ ${ }^{\mathrm{c}}$ University of Iowa Holden Comprehensive Cancer Center, Iowa City, IA, USA \\ ${ }^{\mathrm{d}}$ Department of Urology, University of Iowa, Iowa City, IA, USA
}

Received 9 November 2018

Accepted 12 January 2019

\begin{abstract}
.
Background: Bacillus Calmette-Guerin (BCG) toxicities have been reported but with relative agranularity regarding severity and temporal changes during therapy. In its most severe form, BCG intolerance remains poorly understood and exploring ways to optimize toleration of the most effective treatment (BCG) is prudent.

Objective: To report the results of both patient- and physician-centered metrics of BCG toxicity as part of the National Phase II BCG/Interferon (IFN) study, as well as the efficacy of low-dose BCG/IFN for previously deemed BCG intolerant patients. Methods: Patients were treated with 6 weekly treatments of BCG (various dosing based on prior BCG exposure) with 50 million units of IFN. BCG intolerance was strictly defined. Maintenance BCG was instituted if disease free. Treatment tolerance during induction was measured using physician- and patient-completed evaluations. Linear mixed effects regression was used to evaluate differences.

Results: 533 BCG naïve, 415 BCG failure, and 37 BCG intolerant patients were enrolled. There was no significant difference in quality of life scores between groups $(p=0.70)$. A predictable temporal toxicity pattern was identified in all groups with symptom resolution by day 2-3. Despite BCG intolerant patients having significantly worse bladder pain/spasms and cystitis, treatment discontinuation was under $4 \%$. Recurrence-free survival was similar between naïve and intolerant patients.

Conclusions: BCG toxicity is common and follows a predictable pattern. While at slightly increased risk of toxicity, BCG intolerant patients may be managed successfully with 1/10th dose BCG with IFN.
\end{abstract}

Keywords: Urinary bladder neoplasms, mycobacterium bovis, immunotherapy

\section{INTRODUCTION}

Intravesical Bacillus Calmette-Guerin (BCG) remains the standard of care $[1,2]$ for patients

\footnotetext{
*Correspondence to: Michael A. O’Donnell, MD, Department of Urology, University of Iowa, 200 Hawkins Dr., 3231, RCP, Iowa City, IA 52242-1089, USA. Tel.: +01 319353 8939; Fax: +01 319 356 3900; E-mail: Michael-Odonnell@uiowa.edu.
}

with high-risk, and an option for patients with intermediate-risk, non-muscle invasive bladder cancer (NMIBC) after complete transurethral resection. BCG has shown clear superiority over single agent intravesical chemotherapy in patients without prior BCG treatment [3-8].

Side effects of therapy are typically mild and selflimited, but it has been estimated that $5-10 \%$ of 
patients are unable to complete induction therapy as a result of toxicity $[9,10]$. Prior studies evaluating treatment toxicity have reported findings in a binary fashion (presence vs. absence) which occur at any time during therapy $[10,11]$. Further, symptom severity, outside of the need for treatment delay/cessation, is not reported. To our knowledge, no BCG toxicity assessments have been sufficiently granular to allow analysis of temporal changes in the severity of symptoms during therapy. In addition, most evaluations have been completed from a physician-perspective. Minimal patient-centered evaluations of BCG therapy have been reported [12].

In the National Phase 2 BCG-Interferon- $\alpha$ 2B (IFN) study [13], BCG toxicities were evaluated using both physician- and patient-derived metrics. We now report the results of treatment toxicity in patients treated with combination BCG/IFN, as well as the outcomes of patients previously deemed BCG intolerant.

\section{MATERIALS AND METHODS}

\section{Study population}

Institutional Review Board approval was obtained. The large, over 1,100 patient database created as part of the National Phase 2 BCG/IFN study was queried. Enrollment for this study was extremely liberal and included patients with primary or recurrent NMIBC, with or without carcinoma in situ (CIS), of which some had received prior chemotherapy and/or BCG treatment.

\section{Study treatment}

As previously reported [13], treatment commenced 3-8 weeks after either transurethral resection of bladder tumor (TURBT) or confirmatory cystoscopy, biopsy, or positive cytology. Patients were treated with 6 weekly treatments of BCG (full dose for BCG naïve patients, $1 / 3$ dose for patients with prior BCG failure but not intolerance, and 1/10 dose for those meeting criteria for BCG intolerance) of either TICE (Organon Teknika, Roseland, NJ) or Connaught (Aventis-Pasteur, Swiftwater, PA) strain BCG mixed directly with 50 million units (MU) IFN (Intron A, Schering-Plough, Kenilworth, NJ). Dwell time for each instillation was recorded. BCG intolerance was strictly defined as a history of debilitating cystitis for $>2$ weeks, $\geq 2$ episodes of gross hematuria requiring intervention, inability to hold BCG for $>30$ minutes, repeated severe but limited reactions to BCG or other serious BCG related symptoms excluding actual BCG infection.

Patients who experienced grade 3 toxicity by Toxicity Tolerance Evaluation (TTE) were eligible for a $\sim 1 / 3$ dose reduction (e.g. 1/3rd, 1/10th, 1/30th) from the prior dose after a 2 -week rest period. Additional 2-week treatment delays were allowed for repeat episodes of intolerance, as long as the entire induction cycle was completed within 10 weeks of initiation. The decision to perform dose reduction or simple treatment delay was at the discretion of the treating physician.

Patients began surveillance 4-6 weeks after induction and quarterly for the first 2 years, biannually for years $3-4$, then annually thereafter. All patients without recurrence received reduced dose maintenance therapy consisting of 3-week mini cycles at 3,9 , and 15 months after the end of the induction cycle. Recurrence-free survival was defined as no evidence of visible tumor (unless histologically confirmed to be benign), no positive bladder biopsies or definitively positive urine cytologies, or diagnosis of urothelial carcinoma in other locations (i.e. upper tract, prostate, urethra, or metastatic).

\section{Measures of toxicity}

\section{Quality of Life (QOL) Index}

The QOL Index is a validated questionnaire first published by Bohle [12] evaluating various aspects of how a patient views his/her life. The questionnaire was completed prior to and after completing induction therapy. The questionnaire consists of 4 parts: 1) a satisfaction survey assessing level of happiness in various physical, social, and psychosocial aspects of life rated from 'very satisfied' (1) to 'very dissatisfied' (5), 2) a health survey assessing how patients perception of health affected his/her mood and life rated from 'not at all' (1) to 'a great deal' (5), 3) a patient rating of his/her general physician condition rated from 'very poor' (1) to 'excellent' (7), and 4) patient rating of his/her general quality of life rated from 'very poor' (1) to 'excellent' (7).

\section{Quantitative Symptom Score (QSS)}

The QSS was a patient-completed evaluation used to evaluate the severity of BCG/IFN toxicity during the week following instillation. A QSS survey was completed immediately prior to a patient's weekly BCG instillation (pre-day 0), the evening after his/her instillation (post-day 0), and daily thereafter for 
6 days. The survey specifically asked patients to rate the severity of common BCG toxicities (cystitis, hematuria, fevers \& chills, flu-like symptoms, other adverse events). Severity was rated as none, mild, moderate, or severe, which correlated with a numerical scale of $0-3$.

\section{Tolerance Toxicity Evaluation}

The TTE was a physician-completed evaluation of patient-reported toxicity experienced after each instillation. This was completed by the treating physician 1 week after each instillation (at the time of the next instillation). The survey asked physicians to rate the severity of common BCG toxicities (cystitis, hematuria, fevers \& chills, flu-like symptoms, arthritis \& arthralgias, and other adverse events) in the context of need for treatment. Severity was rated as none, mild, moderate, moderate-severe, severe, or very severe which correlated with a numerical scale of $0-5$. Patients with none or mild symptoms (score 0-1) required no intervention. Moderate symptoms (score 2) required treatment with pharmaceuticals (i.e. non-steroidal anti-inflammatories, anti-spasmodics). Moderate-severe symptoms (score 3) required a treatment delay or dose reduction. Severe symptoms (score 4) lead to BCG cycle cessation while very severe symptoms (score 5) was applied whenever the physician felt any further BCG was strictly contraindicated.

\section{Analysis}

Group differences in quality of life, quantitative symptom scores, and tolerance toxicity evaluation scores over time were assessed using linear mixed effects regression models assuming a compound symmetry covariance structure. In each model, group, time point (pre/post-treatment or cycle number) and an interaction term were included. $P$-values for the interaction term, indicative of whether the rate of change over time differs between patient groups, are reported. For the analysis of symptoms, only measurements collected on day 1 of each cycle were used to determine whether group differences in initial peaking of symptoms after treatment was evidenced. Estimated means and 95\% confidence intervals (CIs) were plotted. Survival probabilities were estimated and plotted using the Kaplan-Meier method. All statistical testing was two-sided using SAS v9.4 (SAS Institute, Cary, NC). Significance was assessed at the $1 \%$ level due to the larger sample size and number of repeated measurements per patient.

\section{RESULTS}

\section{Patient characteristics}

Patient characteristics are listed in Table 1. Five hundred thirty-three BCG naïve (BCG-N) patients $(411 / 533,77.4 \%$ male) with median age of 69 years, 415 BCG failure (BCG-F) patients without prior intolerance $(298 / 415,72.0 \%$ male) with median age of 72 , and 37 BCG intolerant (BCG-I) patients (33/37, $89.2 \%$ male) with median age of 72 years were identified. Median follow-up for all BCG-N, BCG-F, and BCG-I patients was 15.2 months (range 1.1-52.7 months), 9.8 months (range 0.1-49.4 months), and 18.9 months (range 2.3-46.7 months), respectively. Median follow-up among responders for BCG-N, BCG-F, and BCG-I was 26.7 months (range 1.4-52.7 months), 23.1 months (range 0.7-49.4 months), and 22.2 months (range 2.6-46.7 months), respectively. Supplementary Table 1 details the number of each survey returned.

\section{Intolerance measures}

\section{$Q O L$}

Table 2 details the mean pre- and post-treatment QOL scores. Patients in all groups tended to rate general physical condition and general quality of life as poor at baseline with minimal improvement after therapy. There was no significant difference in the change from pre- to post-treatment overall QOL scores between groups $(p=0.70)$. Similarly, there were no significant differences in the subsections of the survey.

\section{QSS}

Figure 1 demonstrates the weekly and weekto-week variation in QSS scores during induction therapy. Symptoms followed a predictable pattern surrounding each instillation. The worst symptoms were noted on day 0 post-instillation with improvement in symptoms to near baseline levels by day 2-3 (Fig. 2). Frequency, urgency, and dysuria were the worst symptoms reported and moderate in severity. BCG-I patients tended to have more severe symptoms than BCG-N and BCG-F patients but only bladder pain/spasms showed a significant difference, demonstrating a possible cumulative effect over time. Urinary urgency showed a trend toward significance. The remainder of the symptoms were not different between groups. 
Table 1

Baseline characteristics of patients treated with BCG plus Interferon induction therapy

\begin{tabular}{|c|c|c|c|}
\hline Variable & Naïve $\mathrm{N}=533(\%)$ & Failure $\mathrm{N}=415(\%)$ & Intolerant $\mathrm{N}=37(\%)$ \\
\hline Median Age (years) & 69 & 72 & 72 \\
\hline \multicolumn{4}{|l|}{ Sex } \\
\hline Female & $120(22.6)$ & $116(28.1)$ & $4(10.8)$ \\
\hline Male & $411(77.4)$ & $298(72.0)$ & $33(89.2)$ \\
\hline \multicolumn{4}{|l|}{ Age } \\
\hline$<65$ & $181(34.0)$ & $109(26.3)$ & $9(24.3)$ \\
\hline $65+$ & $351(66.0)$ & $306(73.7)$ & $28(75.7)$ \\
\hline \multicolumn{4}{|l|}{ Race } \\
\hline White & $497(96.1)$ & $393(96.8)$ & $33(89.2)$ \\
\hline Non-White & $20(3.9)$ & $13(3.2)$ & $4(10.8)$ \\
\hline \multicolumn{4}{|l|}{ TURBTs } \\
\hline$<3$ & 407 (77.4) & $118(28.9)$ & $10(27.0)$ \\
\hline $3+$ & $119(22.6)$ & $290(71.1)$ & $27(73.0)$ \\
\hline \multicolumn{4}{|l|}{ Duration } \\
\hline 0-2 Years & $430(86.3)$ & $226(59.8)$ & $18(51.4)$ \\
\hline $2+$ Years & $68(13.7)$ & $152(40.2)$ & $17(48.6)$ \\
\hline \multicolumn{4}{|l|}{ Prior BCG } \\
\hline Naive & $533(100)$ & $0(0)$ & $0(0)$ \\
\hline$\leq 1$ & $0(0)$ & $255(61.9)$ & $21(56.8)$ \\
\hline$\overline{2}+$ & $0(0)$ & $157(38.1)$ & $16(43.2)$ \\
\hline \multicolumn{4}{|l|}{ Past BCG } \\
\hline No & $533(100)$ & $0(0)$ & $0(0)$ \\
\hline Yes & $0(0)$ & $415(100)$ & $37(100)$ \\
\hline \multicolumn{4}{|l|}{ Prior Maintenance } \\
\hline No & $533(100)$ & 265 (63.9) & $25(67.6)$ \\
\hline Yes & $0(0)$ & $150(36.1)$ & $12(32.4)$ \\
\hline \multicolumn{4}{|l|}{ Stage } \\
\hline CIS & $103(20.4)$ & $116(29.7)$ & 8 (21.6) \\
\hline $\mathrm{T} 1$ & $156(31.0)$ & $74(19.0)$ & $5(13.5)$ \\
\hline $\mathrm{Ta}$ & 245 (48.6) & $200(51.3)$ & $24(64.9)$ \\
\hline \multicolumn{4}{|l|}{ Grade } \\
\hline Low & $78(16.7)$ & $83(22.6)$ & $8(22.2)$ \\
\hline Intermediate & $154(33.0)$ & $104(28.3)$ & $16(44.4)$ \\
\hline High & $234(50.2)$ & $180(49.0)$ & $12(33.3)$ \\
\hline \multicolumn{4}{|l|}{ Tumor } \\
\hline CIS & 49 (9.9) & $86(22.6)$ & 7 (19.4) \\
\hline Solitary & $218(44.0)$ & $99(26.1)$ & $13(36.1)$ \\
\hline$>1$ Tumor & $228(46.1)$ & $195(51.3)$ & $16(44.4)$ \\
\hline \multicolumn{4}{|l|}{ Tumor Size } \\
\hline$<0.5 \mathrm{~cm}$ & $56(12.6)$ & $58(17.5)$ & $5(14.7)$ \\
\hline $0.5-5 \mathrm{~cm}$ & 335 (75.3) & 257 (77.6) & $27(79.4)$ \\
\hline$>5 \mathrm{~cm}$ & $54(12.1)$ & $16(4.8)$ & $2(5.9)$ \\
\hline
\end{tabular}

TTE

Figure 3 shows the week-to-week variation in TTE scores during induction therapy. Symptoms scores tended to be stable across all induction treatments and severity was mild, at worst, in nearly all measures. Two exceptions to this were identified. First, cystitis, while not statistically different, did gradually increase during induction and peaked at mild-moderate in BCG-I patients. Second, BCG-I patients demonstrated a statistically significant difference in fever/chills severity $(p=0.01)$. Table 3 details the specific scores for each measure.

\section{BCG Dwell Time}

The duration that BCG/IFN was able to be held by the patients was similar at the time of first instillation. Though, BCG-I patients were unable to hold the medication as long for the remaining instillations $(p=0.02$, Fig. 4).

\section{Intolerance management and discontinuation}

Pharmaceutical interventions for moderate toxicities captured on TTE (score 2) was performed in 139/437 (31.8\%) BCG-N, 123/341 (36.2\%) BCG-F, 
Table 2

Quality of life scores before and after BCG plus Interferon induction therapy

\begin{tabular}{llccc}
\hline Scale & Group & \multicolumn{2}{c}{ Estimated Mean } & $\begin{array}{c}\text { Interaction } \\
p \text {-value }\end{array}$ \\
\cline { 2 - 4 } General Physical Condition Item & Failure & 2.76 & 2.87 & 0.10 \\
& Intolerant & 2.80 & 3.37 & \\
General QOL Item & Naïve & 2.75 & 2.89 & \\
& Failure & 2.23 & 2.40 & 0.28 \\
& Intolerant & 2.25 & 2.71 & \\
Health Subscale & Naïve & 2.33 & 2.47 & \multirow{2}{*}{0.03} \\
& Failure & 8.23 & 8.45 & \\
Satisfaction Subscalc & Intolerant & 9.13 & 9.09 & 0.49 \\
& Naïve & 8.52 & 8.10 & \\
Overall QOL & Failure & 37.70 & 39.03 & \multirow{2}{*}{0.70} \\
& Intolerant & 40.56 & 39.57 & \\
& Naïve & 36.79 & 38.27 & \\
& Failure & 50.89 & 52.77 & \\
& Intolerant & 54.79 & 54.64 & \\
& Naïve & 50.37 & 51.75 & \\
\hline
\end{tabular}

Table 3

Toxicity tolerance evaluation scores in patients treated with BCG plus Interferon induction therapy

\begin{tabular}{|c|c|c|c|c|c|c|c|}
\hline \multirow[b]{2}{*}{ Symptom } & \multicolumn{6}{|c|}{ EstimatedScoreMean } & \multirow{2}{*}{$\begin{array}{c}\text { Interaction } \\
P \text {-value }\end{array}$} \\
\hline & $\mathrm{Tl}$ & T2 & T3 & $\mathrm{T} 4$ & TS & T6 & \\
\hline \multicolumn{8}{|c|}{ Arthritis/ Arthalgia } \\
\hline Failure & 0.33 & 0.38 & 0.34 & 0.31 & 0.34 & 0.32 & 0.93 \\
\hline Intolerant & 0.45 & 0.63 & 0.54 & 0.63 & 0.52 & 0.54 & \\
\hline Naive & 0.26 & 0.31 & 0.30 & 0.28 & 0.26 & 0.24 & \\
\hline \multicolumn{8}{|l|}{ Cystitis } \\
\hline Failure & 0.54 & 0.66 & 0.70 & 0.67 & 0.67 & 0.66 & 0.88 \\
\hline Intolerant & 0.79 & 0.88 & 0.96 & 0.90 & 0.93 & 1.18 & \\
\hline Naive & 0.38 & 0.51 & 0.49 & 0.51 & 0.46 & 0.50 & \\
\hline \multicolumn{8}{|l|}{ Fever/Chills } \\
\hline Failure & 0.19 & 0.25 & 0.21 & 0.14 & 0.15 & 0.12 & 0.01 \\
\hline Intolerant & 0.87 & 0.51 & 0.58 & 0.45 & 0.25 & 0.55 & \\
\hline Naive & 0.05 & 0.16 & 0.12 & 0.11 & 0.12 & 0.08 & \\
\hline \multicolumn{8}{|c|}{ Flu-like Symptoms } \\
\hline Failure & 0.29 & 0.28 & 0.27 & 0.32 & 0.26 & 0.18 & 0.26 \\
\hline Intolerant & 0.67 & 0.64 & 0.74 & 0.72 & 0.57 & 0.73 & \\
\hline Naive & 0.13 & 0.25 & 0.24 & 0.24 & 0.18 & 0.18 & \\
\hline \multicolumn{8}{|l|}{ Hematuria } \\
\hline Failure & 0.18 & 0.33 & 0.33 & 0.30 & 0.25 & 0.21 & 0.07 \\
\hline Intolerant & 0.17 & 0.38 & 0.31 & 0.24 & 0.23 & 0.39 & \\
\hline Naive & 0.10 & 0.16 & 0.16 & 0.20 & 0.18 & 0.19 & \\
\hline \multicolumn{8}{|l|}{ Other } \\
\hline Failure & 0.35 & 0.39 & 0.32 & 0.38 & 0.34 & 0.29 & 0.59 \\
\hline Intolerant & 0.26 & 0.40 & 0.30 & 0.31 & 0.15 & 0.24 & \\
\hline Naive & 0.25 & 0.23 & 0.26 & 0.33 & 0.30 & 0.25 & \\
\hline
\end{tabular}

$\mathrm{T}=$ Treatment. Severity Score: $0=$ None. 1 = Mild. 2 - Moderate. 3 - Moderate-Severe. 4 - Severe. 5 - Very Severe.

and 14/28 (50\%) BCG-I patients. Treatment delay or dose reduction (score 3 ) was performed in $46 / 437$ (10.5\%) BCG-N, 58/341 (17.1\%) BCG-F, and 5/28 (17.9\%) BCG-I patients. Discontinuation of induction therapy (score 4 or 5 ) was required in $15 / 437$ (3.4\%) BCG-N, 18/341 (5.3\%) BCG-F, and 1/28 (3.6\%) BCG-I patients.

\section{Treatment efficacy}

Recurrence-free survival (RFS) in the intolerant patients was $97 \%$ (CI 82-100\%) at 3 months, $70 \%$ (CI 52-83\%) at 1 year, and 54\% (CI 35-70\%) at 2 years after initiating induction (Fig. 5). This is similar to naïve patient rates of $81 \%$ (CI 78-85\%), 67\% (CI 


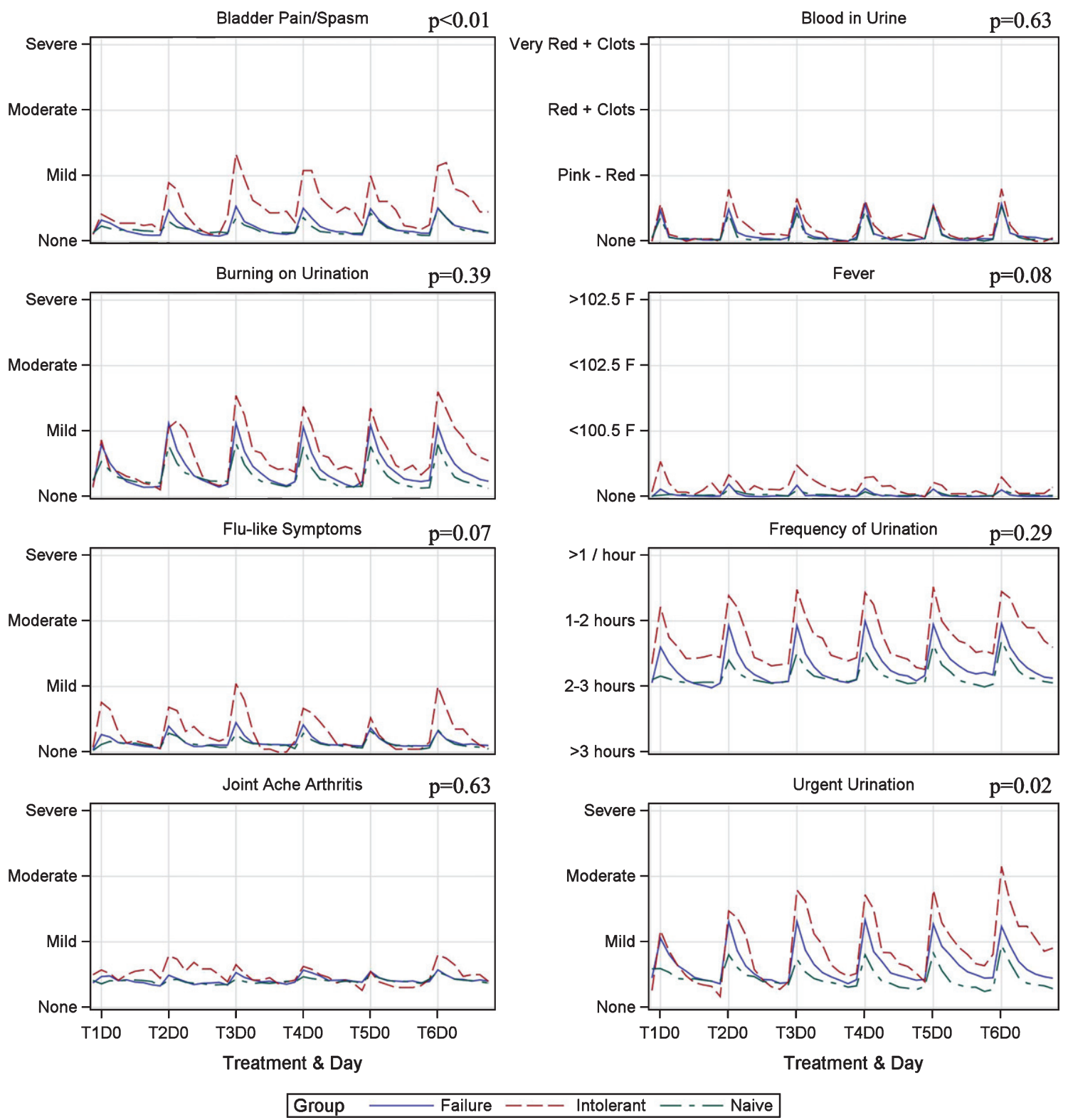

Fig. 1. Quantitative symptom score variation during induction in patients treated with BCG plus Interferon.

62-71\%), and 59\% (CI 64-63\%), respectively, and better than BCG failure but not intolerant patient rates of $80 \%$ (CI 75-83\%), 55\% (CI 49-59\%), and 45\% (CI 40-50\%), respectively.

\section{DISCUSSION}

Side effects at any point during intravesical BCG therapy are common with most being mild, selflimited $^{10,11}$. Though, there has been little dedicated study to the temporal relationship between treatment and toxicities, as well as patient-centered metrics to better understand the side effects of treatment.

Bohle et al. previously described the changes in common treatment toxicities during BCG induction therapy in a limited group of 30 patients by means of a daily questionnaire ${ }^{12}$. Using a different patientcentered evaluation, we identified a similar pattern of treatment toxicities with the worst symptoms being experienced immediately after therapy and abating to near baseline levels after 2-3 days. Frequency, 


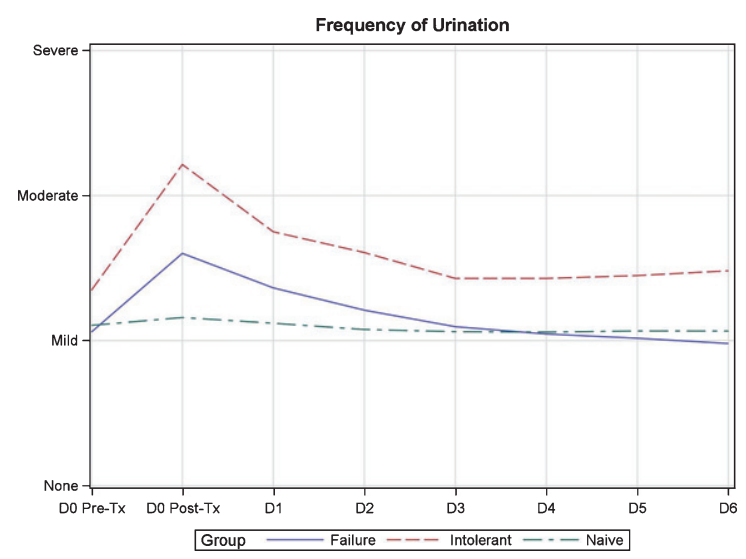

Fig. 2. Quantitative symptom score variation during one week of induction in patients treated with BCG plus Interferon.

urgency, and dysuria were the worst symptoms and noted to be moderate in severity. Patients were also observed to have no significant change in the perceived QOL after BCG induction. Though, it was noted that patients categorized their general physical condition and quality of life as poor at baseline. The etiology of this perception remains unclear. These findings may provide physicians with improved data to counsel patients regarding side effect duration, severity, and the need for medical therapy for toxicity.

The physician-centered evaluation found stable mild toxicity in all domains across all treatments. This appeared to corroborate the patient-centered findings within the fever/chills, flu-like symptoms, arthritis, and hematuria domains. The one domain that was more difficult to compare was cystitis. In order to avoid medical terminology and better evaluate the particular elements of cystitis, patients were specifically asked about bladder pain/spasms, dysuria, frequency and urgency. While the physician-centered metric demonstrated a mild increase in scores during induction, particularly in the BCG intolerant group, the rate of change between groups was not statistically significant and overall remained mild. In the patient-centered evaluation, bladder pain/spasms and dysuria remained mild but frequency and urgency of urination both demonstrated worsening severity as induction proceeded. The exact reason for the disparity is unclear but could be accounted for by a number of factors, including recall bias or lack of granularity in the physician-centered evaluation. Given our findings, cystitis symptoms may be under appreciated by physician evaluation alone. Patients may benefit from keeping a symptom dairy and reviewing these findings with the treating urologist to identify ways to reduce treatment toxicity. Further study of these disparities may aid in better understanding intolerance and the development of future metrics to improve the tolerance of therapy.

With respect to recurrence, the superiority of treatment in BCG naive patients as compared to BCG failure patient has previously been reported [13]. In our study, BCG intolerant patients demonstrated similar RFS as compared to BCG naïve patients. Though, given the small group size, conclusions regarding intolerant patients must be tempered. Prior reports have identified that dilution to $1 / 6$ dose BCG results in a trend toward statistical RFS inferiority [14], as compared to $1 / 3$ dose which has shown sustained efficacy [15]. To our knowledge, this is the first report to demonstrate sustained efficacy of BCG beyond 1/6 the standard dose. Though, it should be noted that the prior studies used BCG as monotherapy while our study utilizes BCG in combination with IFN. The exact mechanism of this improvement is unclear but may be related to the concomitant instillation of IFN.

Our results also demonstrated that BCG intolerant patients follow a similar temporal side effect pattern but tend to have more severe symptoms than BCG naïve and BCG failure patients, even with a reduced dose; though, only the severity of bladder pain/spasms was significantly different. Dose reduction has been explored as a means to improving treatment tolerance. Low dose (1/3 standard dose) BCG monotherapy has demonstrated an improved side effect profile with similar RFS in a mixed cohort of BCG naive and BCG failure patients [15]. Further dilution to $1 / 6$ dose did show similar toxicity but, as noted above, proved less effective [14]. The addition of IFN- $\alpha$ to BCG has shown to lead to immunostimulation by means of IFN- $\gamma$ production [16] and maintains elevated IFN- $\gamma$ levels even with reduced dose BCG [17]. Side effects related to variable dosing of BCG/IFN have been reported but only in small numbers [18]. Most investigation has utilized $50 \mathrm{MU}$, a low dose relative to other studies [19, 20], based on pre-clinical findings [17]. The only other large study involving BCG with IFN demonstrated a higher rate of fever and constitutional symptoms with IFN and no improvement in RFS as compared to BCG monotherapy in a BCG naïve population. Though, in that study, IFN was used with full dose BCG and further granularity of treatment tolerance/toxicity was not reported [21].

BCG intolerance remains an understudied subpopulation of NMIBC patients. The European Association of Urology (EAU) defines BCG 

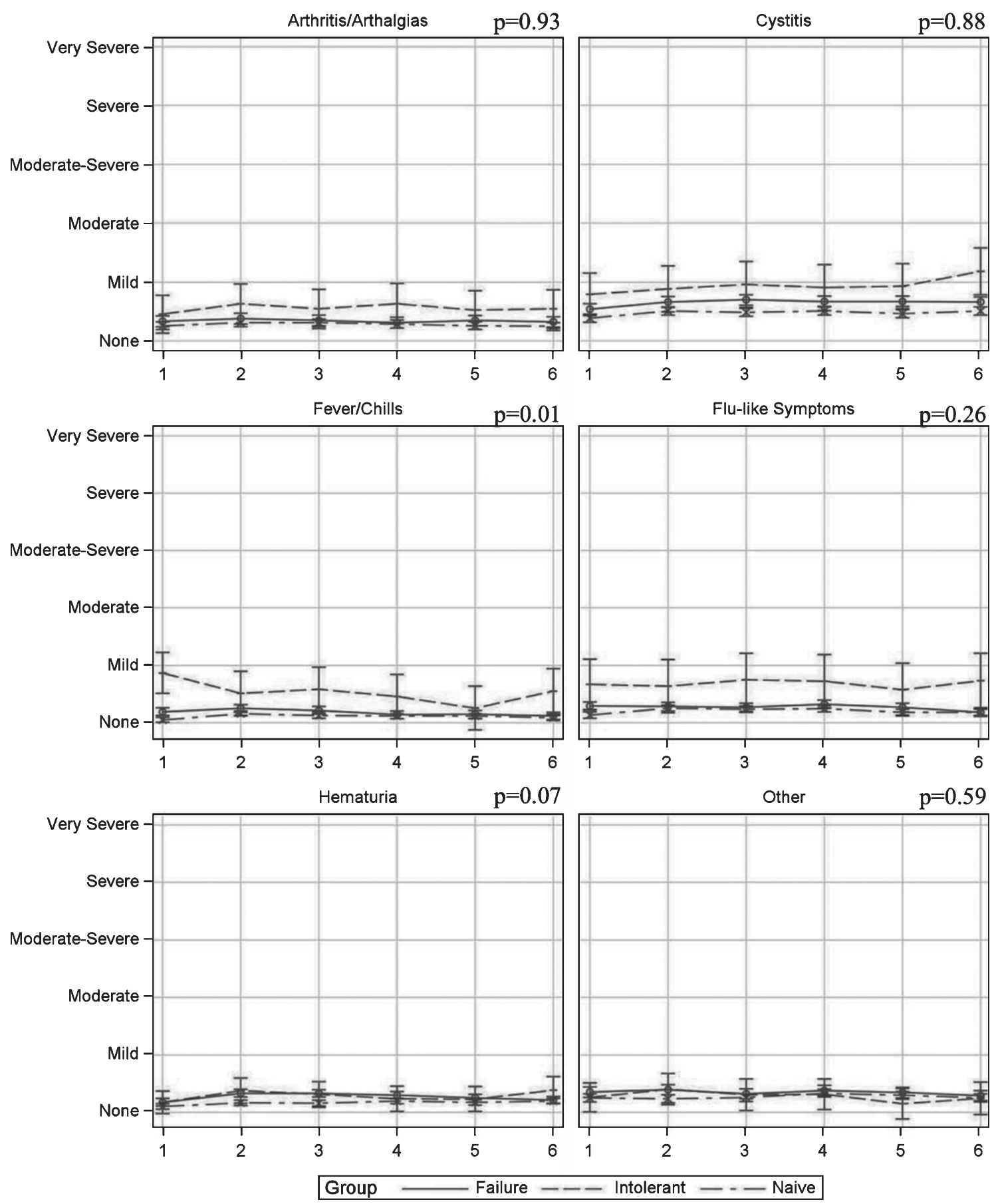

Fig. 3. Toxicity tolerance evaluation score variation during induction in patients treated with BCG plus Interferon induction therapy.

intolerance as "severe side effects that prevent further BCG instillation before completing induction" and goes on to note that "little is known about the optimal treatment in patients with high-risk tumours who could not complete BCG instillations because of intolerance" [1]. The American Urological Association (AUA) has no formal definition for intolerance [2]. Neither organization has recommendations for therapy in those deemed intolerant. An improved understanding of BCG toxicity is needed to (1) 
consider interventions (i.e. medication) which may lessen treatment toxicity and (2) better define what truly constitutes BCG intolerance.

There are a number of limitations to our study. First, the study was not powered to directly answer our question, but this is offset by the large overall study size. Second, the small size of the intolerant arm limits our ability to more thoroughly analyze this cohort. Third, the variable dosing regimen used makes direct comparative efforts difficult. Further, there was no BCG monotherapy arm in this study and thus direct comparison of BCG+IFN tolerance to BCG cannot be made. Though, the rates of local and systemic side effects, as well as treatment discontinuation, are similar to those previously reported [11]. It is the authors opinion that these findings are analogous to the expected results of patients treated with BCG monotherapy, particularly in the BCG failure and BCG intolerant groups. Further investigation is needed to validate this theory. The strict definition of what constituted BCG intolerance was arbitrarily determined by the primary investigator. This may have limited the number of appropriate patients enrolled in the BCG intolerance arm. Though, as there are no agreed upon definitions by the governing urologic organizations, this was felt to be acceptable. Only one of the surveys utilized in this study was validated in a NMIBC patient cohort, thus leading to possible measurement error. Though, our study

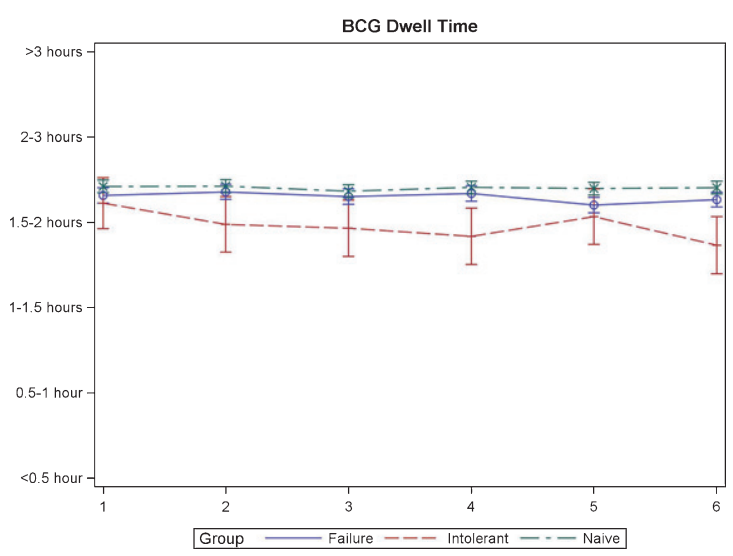

Fig. 4. BCG dwell time during induction in patients treated with BCG plus Interferon.

does include multiple surveys and considers multiple perspectives of BCG toxicity. Not all surveys were returned/completed (Supplementary Table 1) which also limits the ability to generalize our results. Finally, the study did not collect data on the rate of progression in patients who failed. Thus, we cannot report on this important variable.

\section{CONCLUSIONS}

Patient-centered evaluation of BCG/IFN treatment identified that side effects are common, tend to follow

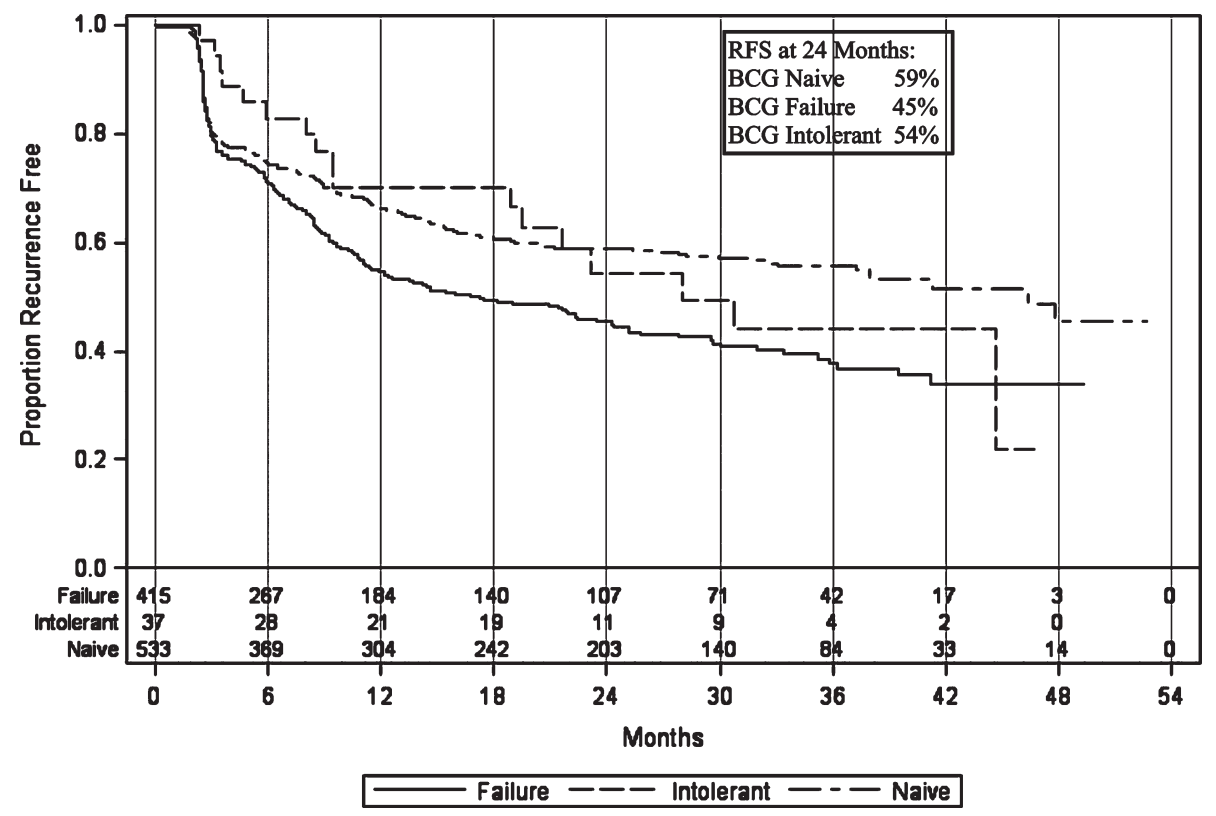

Fig. 5. Recurrence-free survival in patients treated with BCG plus Interferon therapy. 
a predictable pattern, and do not significantly change a patient's quality of life. Physician-centered metrics corroborate most of the patient-reported findings with the exception of cystitis. Finally, 1/10th dose BCG with IFN appears to be effective in patients previously deemed BCG intolerant. Further understanding of the patient experience during intravesical therapy and dedicated study of intolerance to BCGbased therapies is warranted to better address ways to overcome toxicities.

\section{CONFLICT OF INTEREST}

M O'Donnell reports the following disclosures:

- Abbott Molecular (Grant Support)

- Fidia Farmaceutica (Consultant)

- Medical Enterprises (Consultant; Investigator)

- PhotoCure (Investigator)

- Theralase (Consultant)

- Urogen (Consultant; Investigator)

- Vaxiion (Consultant)

- R Steinberg, L Thomas, S Mott have no conflict of interest to report

\section{SUPPLEMENTARY MATERIAL}

The supplementary material is available in the electronic version of this article: http://dx. doi.org/10.3233/BLC-180203.

\section{REFERENCES}

[1] Babjuk M, Bohle A, Burger M, Capoun O, Cohen D, Comperat EM, et al. EAU Guidelines on Non-Muscle-invasive Urothelial Carcinoma of the Bladder: Update 2016. European Urology. 2017;71(3):447-61.

[2] Chang SS, Boorjian SA, Chou R, Clark PE, Daneshmand S, Konety BR, et al. Diagnosis and Treatment of Non-Muscle Invasive Bladder Cancer: AUA/SUO Guideline. J Urol. 2016;196(4):1021-9.

[3] Brosman SA. Experience with bacillus Calmette-Guerin in patients with superficial bladder carcinoma. J Urol. 1982;128(1):27-30.

[4] Lamm DL, Blumenstein BA, Crawford ED, Montie JE, Scardino P, Grossman HB, et al. A randomized trial of intravesical doxorubicin and immunotherapy with bacille Calmette-Guerin for transitional-cell carcinoma of the bladder. The New England Journal of Medicine. 1991;325(17):1205-9.

[5] Lamm DL, Blumenstein BA, David Crawford E, Crissman JD, Lowe BA, Smith JA Jr, et al. Randomized intergroup comparison of bacillus calmette-guerin immunotherapy and mitomycin $\mathrm{C}$ chemotherapy prophylaxis in superficial transitional cell carcinoma of the bladder a southwest oncology group study. Urologic Oncology. 1995;1(3):119-26.
[6] Sylvester RJ, Brausi MA, Kirkels WJ, Hoeltl W, Calais Da Silva F, Powell PH, et al. Long-term efficacy results of EORTC genito-urinary group randomized phase 3 study 30911 comparing intravesical instillations of epirubicin, bacillus Calmette-Guerin, and bacillus Calmette-Guerin plus isoniazid in patients with intermediate- and high-risk stage Ta T1 urothelial carcinoma of the bladder. European Urology. 2010;57(5):766-73.

[7] Sylvester RJ, van der Meijden AP, Witjes JA, Kurth K. Bacillus calmette-guerin versus chemotherapy for the intravesical treatment of patients with carcinoma in situ of the bladder: A meta-analysis of the published results of randomized clinical trials. J Urol. 2005;174(1):86-91; discussion -2.

[8] Malmstrom PU, Wijkstrom H, Lundholm C, Wester K, Busch C, Norlen BJ. 5-year followup of a randomized prospective study comparing mitomycin $\mathrm{C}$ and bacillus Calmette-Guerin in patients with superficial bladder carcinoma. Swedish-Norwegian Bladder Cancer Study Group. The Journal of Urology. 1999;161(4):1124-7.

[9] van der Meijden AP, Sylvester RJ, Oosterlinck W, Hoeltl W, Bono AV, Group EG-UTC. Maintenance Bacillus CalmetteGuerin for Ta T1 bladder tumors is not associated with increased toxicity: Results from a European Organisation for Research and Treatment of Cancer Genito-Urinary Group Phase III Trial. European Urology. 2003;44(4):42934.

[10] Brausi M, Oddens J, Sylvester R, Bono A, van de Beek C, van Andel G, et al. Side effects of Bacillus Calmette-Guerin (BCG) in the treatment of intermediate- and high-risk $\mathrm{Ta}, \mathrm{T} 1$ papillary carcinoma of the bladder: Results of the EORTC genito-urinary cancers group randomised phase 3 study comparing one-third dose with full dose and 1 year with 3 years of maintenance BCG. European Urology. 2014;65(1):69-76.

[11] Lamm DL, van der Meijden PM, Morales A, Brosman SA, Catalona WJ, Herr HW, et al. Incidence and treatment of complications of bacillus Calmette-Guerin intravesical therapy in superficial bladder cancer. J Urol. 1992;147(3):596-600.

[12] Bohle A, Balck F, von Weitersheim J, Jocham D. The quality of life during intravesical bacillus Calmette-Guerin therapy. J Urol. 1996;155(4):1221-6.

[13] Joudi FN, Smith BJ, O'Donnell MA, National BCGIPIG. Final results from a national multicenter phase II trial of combination bacillus Calmette-Guerin plus interferon alpha-2B for reducing recurrence of superficial bladder cancer. Urologic Oncology. 2006;24(4):344-8.

[14] Ojea A, Nogueira JL, Solsona E, Flores N, Gomez JM, Molina JR, et al. A multicentre, randomised prospective trial comparing three intravesical adjuvant therapies for intermediate-risk superficial bladder cancer: Low-dose bacillus Calmette-Guerin $(27 \mathrm{mg}$ ) versus very low-dose bacillus Calmette-Guerin (13.5mg) versus mitomycin C. European Urology. 2007;52(5):1398-406.

[15] Martinez-Pineiro JA, Flores N, Isorna S, Solsona E, Sebastian JL, Pertusa C, et al. Long-term follow-up of a randomized prospective trial comparing a standard $81 \mathrm{mg}$ dose of intravesical bacille Calmette-Guerin with a reduced dose of $27 \mathrm{mg}$ in superficial bladder cancer. BJU International. 2002;89(7):671-80.

[16] Luo Y, Chen X, Han R, O'Donnell MA. Recombinant bacille Calmette-Guerin (BCG) expressing human interferon-alpha 2B demonstrates enhanced immunogenicity. Clin Exp Immunol. 2001;123(2):264-70. 
[17] Luo Y, Chen X, Downs TM, DeWolf WC, O'Donnell MA. IFN-alpha 2B enhances Th1 cytokine responses in bladder cancer patients receiving Mycobacterium bovis bacillus Calmette-Guerin immunotherapy. Journal of Immunology. 1999;162(4):2399-405.

[18] Stricker P, Pryor K, Nicholson T, Goldstein D, Golovsky D, Ferguson R, et al. Bacillus Calmette-Guerin plus intravesical interferon alpha- $2 \mathrm{~b}$ in patients with superficial bladder cancer. Urology. 1996;48(6):957-62.

[19] Glashan RW. A randomized controlled study of intravesical alpha-2b-interferon in carcinoma in situ of the bladder. J Urol. 1990;144(3):658-61.
[20] Torti FM, Shortliffe LD, Williams RD, Pitts WC, Kempson RL, Ross JC, et al. Alpha-interferon in superficial bladder cancer: A Northern California Oncology Group Study. Journal of clinical oncology : Official journal of the American Society of Clinical Oncology. 1988;6(3):476-83.

[21] Nepple KG, Lightfoot AJ, Rosevear HM, O'Donnell MA, Lamm DL, Bladder Cancer Genitourinary Oncology Study G. Bacillus Calmette-Guerin with or without interferon alpha- $2 \mathrm{~b}$ and megadose versus recommended daily allowance vitamins during induction and maintenance intravesical treatment of nonmuscle invasive bladder cancer. J Urol. 2010;184(5):1915-9. 